\title{
An Intuitionistic Fuzzy Finite Automaton on Homomorphism and Admissible relation
}

\author{
Jency Priya. $\mathrm{K}^{1^{*}}$, Telesphor Lakra ${ }^{2}$, Jeny Jordon. $\mathrm{A}^{3}$, Rajaretnam. $\mathrm{T}^{4}$ \\ 1,2,3,4 (Department of Mathematics, St. Joseph's College (Autonomous), Tiruchirappalli, TN, India)
}

\begin{abstract}
An intuitionistic fuzzy finite automaton with an unique membership transition on an input symbol IFA-UM is considered. IFA-UM homomorphism and strong homomorphism are defined. Admissible relation on the set of states of an IFA-UM is characterized. Each admissible relation on an IFA-UMA, finds an IFA-UM $\mathcal{A}_{1}$ and there is a strong homomorphism from $\mathcal{A}$ to $\mathcal{A}_{1}$.
\end{abstract}

Keywords: Intuitionistic fuzzy finite automaton IFA-UM, Intuitionistic fuzzy behavior, Homomorphism, Strong homomorphism and Admissible relation.

\section{Introduction}

The concept of "Fuzzy Sets" [12] was introduced to describe vagueness mathematically in its abstract form by giving a grade of membership to each member of a given set. L.A. Zadeh thus laid the foundations of fuzzy set theory in 1965. He considers the "membership" in a fuzzy set not as a matter of affirmation or denial, rather one of degree. Over the last five decades, his proposal has gained important recognition in the theory of formal languages. Automata theory is closely related to formal language theory since the automata are often defined by the class of formal languages they are able to recognize. A finite automaton gives a finite representation of a regular language that may be an infinite set. To deal with any imprecision or uncertainty arising out of the fuzziness in modeling some systems, fuzzy automata and fuzzy languages have been introduced [6]. In fuzzy automata, a set of strings recognized is said to be a fuzzy language. In a fuzzy finite state automaton, there may be more than one fuzzy transition from a state on an input symbol with a given membership value as assigned by Santos, Wee and $\mathrm{Fu}[10,11]$. This development was followed by the postulation that there can be at most one transition on an input, which can be constructed equivalently from a fuzzy finite state automaton. This postulation led to the description of deterministic fuzzy finite state automaton by Malik and Mordeson [7]. However, the deterministic fuzzy finite state automaton only acts as a deterministic fuzzy recognizer, whereas the fuzzy regular languages accepted by the fuzzy finite state automaton and deterministic fuzzy finite state automaton need not necessarily be equal (i.e. the degree of a string need not be the same). It is introduced [8] as fuzzy finite state automaton with unique membership transition (uffsa) in which the membership values of any recognized string in both the systems are the same.

The concept intuitionistic fuzzy sets (IFS) introduced by Atanassov [1-3] has been found to be highly useful to deal with vagueness, since the IFS is characterized by two functions expressing the degree of belongingness and the degree of non-belongingness. Burillo and Bustince [4] proved that the notion of vague sets coincides with that of intuitionistic fuzzy sets. This idea is a natural generalization of a standard fuzzy set, and seems to be useful in modeling many real life situations and it is easier describing negative factors than the positive attributes. It is possible to obtain intuitionistic fuzzy language by introducing membership and nonmembership value to the strings of fuzzy language. Jun [5] introduced the intuitionistic fuzzy finite state machines. For any intuitionistic fuzzy finite automaton (IFA) there is an equivalent intuitionistic fuzzy finite automaton with unique membership (IFA-UM) transition on an input symbol [9]. The IFA-UM produces the same membership values for the recognized strings.

In this paper, the behaviors of a homomorphism of an IFA-UM of both membership and nonmembership are discussed. If there is a strong homomorphism and the function $\alpha$ is bijective then their behaviors hold equality. The concept of admissible relation of IFA-UM is introduced and for every IFA-UM $\mathcal{A}$, there is a $\mathcal{A}_{1}$ such that there exists a strong homomorphism between $\mathcal{A}$ and $\mathcal{A}_{1}$ respectively.

\section{Basic definitions}

Definition 2.1 Given a nonempty set $\sum$. Intuitionistic fuzzy sets ( IFS) in $\sum$ is an object having the form $A=\left\{\left(\mathrm{x}, \mu_{A}(\mathrm{x}), v_{A}(\mathrm{x})\right) \mid \mathrm{x} \in \sum\right\}$, where $\mu_{A}: \sum \rightarrow[0,1]$ and $v_{A}: \sum \rightarrow[0,1]$ denote the degree of membership and the degree of non-membership of each element $x \in \sum$ to the set $A$ respectively, and $0 \leq \mu_{A}(\mathrm{x}) \leq v_{A}(\mathrm{x}) \leq 1$ for each $x \in \sum$. For the sake of simplicity, use the notation $A=\left(\mu_{A}, v_{A}\right)$ instead of $A=\left\{\left(\mathrm{x}, \mu_{A}(\mathrm{x}), v_{A}(\mathrm{x})\right) \mid \mathrm{x} \in \sum\right\}$.

Definition 2.2 Intuitionistic fuzzy finite automaton with unique membership transition on an input symbol(IFA$\mathrm{UM})$ is an ordered 5-tuple $\mathcal{A}=(\mathrm{Q}, \Sigma, \mathrm{A}, \mathrm{i}, \mathrm{f})$ where $\mathrm{Q}$ is a finite non-empty set of states, $\Sigma$ is a finite non-empty 
set of input symbols, $A=\left(\mu_{A}, v_{A}\right)$ is an intuitionistic fuzzy subset of $Q \times \Sigma \times Q$, where $\mu_{A}$ and $v_{A}$ denotes the degree of membership and non-membership values respectively such that $\mu_{A}(\mathrm{p}, \mathrm{a}, \mathrm{q})=\mu_{A}\left(\mathrm{p}, \mathrm{a}, \mathrm{q}^{\prime}\right)$ for some $q, q^{\prime} \in Q$ then $q=q^{\prime}, i=\left(\mathrm{i}_{\mu_{A}}, \mathrm{i}_{v_{A}}\right)$ and $f=\left(\mathrm{f}_{\mu_{A}}, \mathrm{f}_{v_{A}}\right)$ are called the intuitionistic fuzzy subset of initial state and final states respectively, from $Q \rightarrow[0,1]$.

Definition 2.3 Let $\mathcal{A}=(\mathrm{Q}, \Sigma, \mathrm{A}, \mathrm{i}, \mathrm{f})$ be an IFA-UM. Define an IFS $A^{*}=\left(\mu_{A}^{*}, v_{A}^{*}\right)$ in $Q \times \Sigma^{*} \times Q$ as follows: $\forall p, q \in Q, x \in \Sigma^{*}, a \in \Sigma$,

$$
\begin{aligned}
& \mu_{A}^{*}(\mathrm{q}, \in, \mathrm{p})=\left\{\begin{array}{ll}
1, & \text { if } p=q \\
0, & \text { if } p \neq q
\end{array} \text {, and } \quad v_{A}^{*}(\mathrm{q}, \in, \mathrm{p})=\left\{\begin{array}{ll}
0, & \text { if } p=q \\
1, & \text { if } p \neq q
\end{array},\right.\right. \\
& \mu_{A}^{*}(\mathrm{q}, \mathrm{xa}, \mathrm{p})=\vee\left\{\mu_{A}^{*}(\mathrm{q}, \mathrm{x}, \mathrm{r}) \wedge \mu_{A}(\mathrm{r}, \mathrm{a}, \mathrm{p}) \mid \mathrm{r} \in Q\right\}, \\
& v_{A}^{*}(\mathrm{q}, \mathrm{xa}, \mathrm{p})=\wedge\left\{v_{A}^{*}(\mathrm{q}, \mathrm{x}, \mathrm{r}) \vee v_{A}(\mathrm{r}, \mathrm{a}, \mathrm{p}) \mid \mathrm{r} \in Q\right\} .
\end{aligned}
$$

Definition 2.4 The intuitionistic fuzzy behavior of IFA-UM is $\mathrm{L}_{\mathcal{A}}=\left(\mathrm{L}_{\mu_{\mathcal{A}}}, \mathrm{L}_{v_{\mathcal{A}}}\right)$

Definition 2.5 A string $x \in \Sigma^{*}$ is recognized by $\mathcal{A}$ if

$$
\begin{aligned}
& \mathrm{L}_{\mu_{\mathcal{A}}}(\mathrm{x})=\vee\left\{\mathrm{i}_{\mu_{\mathrm{A}}}(\mathrm{p}) \wedge \mu_{\mathrm{A}}^{\star}(\mathrm{p}, \mathrm{x}, \mathrm{q}) \wedge \mathrm{f}_{\mu_{\mathrm{A}}}(\mathrm{q}) \mid \mathrm{p}, \mathrm{q} \in \mathrm{Q}\right\}>0 \text { and } \\
& \mathrm{L}_{v_{\mathcal{A}}}(\mathrm{x})=\wedge\left\{\mathrm{i}_{v_{\mathrm{A}}}(\mathrm{p}) \vee v_{\mathrm{A}}^{\star}(\mathrm{p}, \mathrm{x}, \mathrm{q}) \vee \mathrm{f}_{v_{\mathrm{A}}}(\mathrm{q}) \mid \mathrm{p}, \mathrm{q} \in \mathrm{Q}\right\}<1
\end{aligned}
$$

\section{Homomorphism}

Definition 3.1 Let $\mathcal{A}$ and $\mathcal{B}$ be two IFA-UM's. A pair $(\alpha, \beta)$ of mappings $\alpha: Q_{A} \rightarrow Q_{B}$ and $\beta: \Sigma \rightarrow \Gamma$ is called a homomorphism, written $(\alpha, \beta): \mathcal{A} \rightarrow \mathcal{B}$, if $\forall \mathrm{p}, \mathrm{q} \in \mathrm{Q}_{\mathrm{A}}$ and $\forall \mathrm{a} \in \Sigma$ $\mu_{\mathrm{A}}(\mathrm{p}, \mathrm{a}, \mathrm{q}) \leq \mu_{\mathrm{B}}(\alpha(\mathrm{p}), \beta(\mathrm{a}), \alpha(\mathrm{q}))$ and $v_{\mathrm{A}}(\mathrm{p}, \mathrm{a}, \mathrm{q}) \geq v_{\mathrm{B}}(\alpha(\mathrm{p}), \beta(\mathrm{a}), \alpha(\mathrm{q}))$.

(ii) $\quad \forall x \in Q_{A}, i_{\mu_{A}}(p) \leq i_{\mu_{B}}(\alpha(p))$ and $\forall x \in Q_{A}, i_{v_{A}}(p) \geq i_{v_{B}}(\alpha(p))$

(iii) $\quad \forall x \in Q_{A}, f_{\mu_{A}}(p) \leq \mathrm{f}_{\mu_{B}}(\alpha(p))$ and $\forall x \in Q_{A}, f_{v_{A}}(p) \geq \mathrm{f}_{v_{B}}(\alpha(p))$

The pair $(\alpha, \beta)$ is called a strong homomorphism if $\forall p, q \in Q_{A}$ and $\forall a \in \Sigma$,

(a) $\quad \mu_{B}(\alpha(p), \beta(a), \alpha(q))=V\left\{\mu_{A}(p, a, t) \mid t \in Q_{A}, \alpha(t)=\alpha(q)\right\}$ and $v_{B}(\alpha(p), \beta(a), \alpha(q))=\wedge\left\{v_{A}(p, a, t) \mid t \in Q_{A}, \alpha(t)=\alpha(q)\right\}$

Further, if $\alpha(p)=\alpha(q)$ then

(a) $\quad \mu_{\mathrm{B}}(\alpha(\mathrm{p}), \beta(\mathrm{a}), \alpha(\mathrm{q}))=\mathrm{v}\left\{\mu_{\mathrm{A}}(\mathrm{s}, \mathrm{a}, \mathrm{t}) \mid \alpha(\mathrm{t})=\alpha(\mathrm{q}), \alpha(\mathrm{s})=\alpha(\mathrm{p})\right\}$ and $v_{\mathrm{B}}(\alpha(\mathrm{p}), \beta(\mathrm{a}), \alpha(\mathrm{q}))=\Lambda\left\{v_{\mathrm{A}}(\mathrm{p}, \mathrm{a}, \mathrm{t}) \mid \alpha(\mathrm{t})=\alpha(\mathrm{q}), \alpha(\mathrm{s})=\alpha(\mathrm{p})\right\}$

$$
\mathrm{i}_{\mu_{B}}(\alpha(\mathrm{p}))=\vee\left\{\mathrm{i}_{\mu_{A}}(\mathrm{t}) \mid \mathrm{t} \in \mathrm{Q}_{\mathrm{A}}, \alpha(\mathrm{t})=\alpha(\mathrm{p})\right\} \text { and } \mathrm{i}_{v_{B}}(\alpha(\mathrm{p}))=\Lambda\left\{\mathrm{i}_{v_{A}}(\mathrm{t}) \mid \mathrm{t} \in \mathrm{Q}_{\mathrm{A}}, \alpha(\mathrm{t})=\alpha(\mathrm{p})\right\}
$$

A homomorphism (strong homomorphism) $(\alpha, \beta): \mathcal{A} \rightarrow \mathcal{B}$ is an isomorphism (strong isomorphism), if $\alpha$ and $\beta$ are both one-one and onto.

Definition 3.2 If $(\alpha, \beta)$ is a strong homomorphism with $\alpha$ one-one, then $\forall p, q \in Q_{A}$ and $\forall a \in \Sigma$, $\mu_{\mathrm{B}}(\alpha(\mathrm{p}), \beta(\mathrm{a}), \alpha(\mathrm{q}))=\mu_{\mathrm{A}}(\mathrm{p}, \mathrm{a}, \mathrm{q})$ and $v_{\mathrm{B}}(\alpha(\mathrm{p}), \beta(\mathrm{a}), \alpha(\mathrm{q}))=v_{\mathrm{A}}(\mathrm{p}, \mathrm{a}, \mathrm{q})$. If $\Sigma=\Gamma$ and $\beta$ is the identity map, then we write $\alpha: \mathcal{A} \rightarrow \mathcal{B}$ and say that $\alpha$ is a homomorphism or strong homomorphism accordingly.

Example 3.3 Let $Q_{A}=\left\{q_{1}, q_{2}, q_{3}\right\}, \Sigma=\{a, b\}$ with $\mu_{A}: Q_{A} \times \Sigma \times Q_{A} \rightarrow[0,1]$ and $v_{A}: Q_{A} \times \Sigma \times Q_{A} \rightarrow[0,1]$. Let $\mathrm{Q}_{\mathrm{B}}=\left\{\mathrm{q}_{1}^{\prime}, \mathrm{q}_{2}^{\prime}, \mathrm{q}_{3}^{\prime}\right\}, \Gamma=\{\mathrm{a}, \mathrm{b}\}$ with $\mu_{\mathrm{B}}: \mathrm{Q}_{\mathrm{B}} \times \Sigma \times \mathrm{Q}_{\mathrm{B}} \rightarrow[0,1]$ and $v_{\mathrm{B}}: \mathrm{Q}_{\mathrm{B}} \times \Sigma \times \mathrm{Q}_{\mathrm{B}} \rightarrow[0,1]$. The intuitionistic fuzzy initial states with membership values are $\mathrm{i}_{\mu_{\mathrm{A}}}\left(\mathrm{q}_{1}\right)=0.8, \mathrm{i}_{\mu_{\mathrm{B}}}\left(\mathrm{q}_{1}^{\prime}\right)=0.8$ and non-membership values are $\mathrm{i}_{v_{A}}\left(\mathrm{q}_{1}\right)=0.2, \mathrm{i}_{v_{B}}\left(\mathrm{q}_{1}^{\prime}\right)=0.2$. The intuitionistic fuzzy final states with membership values are $\mathrm{f}_{\mu_{A}}\left(\mathrm{q}_{3}\right)=$ $0.7, \mathrm{f}_{\mathrm{H}_{\mathrm{B}}}\left(\mathrm{q}_{3}^{\prime}\right)=0.7$ and non-membership values are $\mathrm{f}_{v_{A}}\left(\mathrm{q}_{1}\right)=0.3, \mathrm{f}_{v_{B}}\left(\mathrm{q}_{3}^{\prime}\right)=0.3$. The intuitionistic fuzzy transitions are given as follows:

$$
\begin{aligned}
& \mu_{A}\left(\mathrm{q}_{1}, \mathrm{a}, \mathrm{q}_{1}\right)=0.3, \mu_{A}\left(\mathrm{q}_{1}, \mathrm{~b}, \mathrm{q}_{2}\right)=0.6, \mu_{A}\left(\mathrm{q}_{2}, \mathrm{a}, \mathrm{q}_{1}\right)=0.3, \\
& \mu_{A}\left(\mathrm{q} 2, \mathrm{~b}, \mathrm{q}_{3}\right)=0.6, \mu_{A}\left(\mathrm{q} 3, \mathrm{~b}, \mathrm{q}_{2}\right)=0.5, \mu_{A}\left(\mathrm{q}_{3}, \mathrm{a}, \mathrm{q}_{3}\right)=0.2 \\
& v_{A}\left(\mathrm{q}_{1}, \mathrm{a}, \mathrm{q}_{1}\right)=0.4, v_{A}\left(\mathrm{q}_{1}, \mathrm{~b}, \mathrm{q}_{2}\right)=0.4, v_{A}\left(\mathrm{q}_{2}, \mathrm{a}, \mathrm{q}_{1}\right)=0.5, \\
& v_{A}\left(\mathrm{q}_{2}, \mathrm{~b}, \mathrm{q}_{3}\right)=0.2, v_{A}\left(\mathrm{q}_{3}, \mathrm{~b}, \mathrm{q}_{2}\right)=0.4, v_{A}\left(\mathrm{q}_{3}, \mathrm{a}, \mathrm{q}_{3}\right)=0.6 \\
& \text { and } \mu_{B}\left(\mathrm{q}_{1}^{\prime}, \mathrm{a}, \mathrm{q}_{1}^{\prime}\right)=0.3, \mu_{B}\left(\mathrm{q}_{1}^{\prime}, \mathrm{b}, \mathrm{q}_{2}^{\prime}\right)=0.6, \mu_{B}\left(\mathrm{q}_{2}^{\prime}, \mathrm{a}, \mathrm{q}_{1}^{\prime}\right)=0.3, \mu_{B}\left(\mathrm{q}_{3}^{\prime}, \mathrm{b}, \mathrm{q}_{2}^{\prime}\right)=0.5, \mu_{B}\left(\mathrm{q}_{3}^{\prime}, \mathrm{a}, \mathrm{q}_{1}^{\prime}\right)=0.5, \\
& v_{B}\left(\mathrm{q}_{1}^{\prime}, \mathrm{a}_{1}^{\prime} \mathrm{q}_{1}^{\prime}\right)=0.4, v_{B}\left(\mathrm{q}_{1}^{\prime}, \mathrm{b}, \mathrm{q}_{2}^{\prime}\right)=0.4, v_{B}\left(\mathrm{q}_{2}^{\prime}, \mathrm{a}, \mathrm{q}_{1}^{\prime}\right)=0.5, v_{B}\left(\mathrm{q}_{3}^{\prime}, \mathrm{b}, \mathrm{q}_{2}^{\prime}\right)=0.4, v_{B}\left(\mathrm{q}_{3}^{\prime}, \mathrm{a}, \mathrm{q}_{1}^{\prime}\right)=0.5 \\
& \text { Let } \alpha: Q_{A} \rightarrow Q_{B} \text { by } \alpha\left(\mathrm{q}_{1}\right)=q_{1}^{\prime}, \alpha\left(\mathrm{q}_{2}\right)=q_{2}^{\prime}, \alpha\left(\mathrm{q}_{3}\right)=q_{3}^{\prime} \text { and } \beta: \Sigma \rightarrow \Gamma \text { by } \beta(\mathrm{a})=\mathrm{a}, \beta(\mathrm{b})=b .
\end{aligned}
$$

Clearly, $(\alpha, \beta)$ is a strong homomorphism from $\mathcal{A}$ into $\mathcal{B}$. Since $\alpha$ and $\beta$ are bijective, $(\alpha, \beta)$ is an isomorphism of IFA-UM. 
Lemma 3.4 Let $\mathcal{A}$ and $\mathcal{B}$ be two IFA-UM's and $(\alpha, \beta)$ be a strong homomorphism, for all $q, r \in Q_{A}, \forall a \in \Sigma$ if $\quad \mu_{B}(\alpha(\mathrm{q}), \beta(\mathrm{a}), \alpha(\mathrm{r}))>0 \quad$ and $\quad v_{B}(\alpha(\mathrm{q}), \beta(\mathrm{a}), \alpha(\mathrm{r}))<1$, then there exists $t \in Q_{A} \quad$ such that $\mu_{A}(\mathrm{q}, \mathrm{a}, \mathrm{t})>0, v_{A}(\mathrm{q}, \mathrm{a}, \mathrm{t})<1$ and $\alpha(\mathrm{t})=\alpha(\mathrm{r})$. Furthermore, for all $p \in Q_{A}$ if $\alpha(\mathrm{p})=\alpha(\mathrm{q})$, then $\mu_{A}(\mathrm{q}, \mathrm{a}, \mathrm{t}) \geq \mu_{A}(\mathrm{p}, \mathrm{a}, \mathrm{r})$ and $v_{A}(\mathrm{q}, \mathrm{a}, \mathrm{t}) \leq v_{A}(\mathrm{p}, \mathrm{a}, \mathrm{r})$.

Proof: Let $p, q, r \in Q_{A}, \forall a \in \Sigma$, then $\mu_{B}(\alpha(\mathrm{q}), \beta(\mathrm{a}), \alpha(\mathrm{r}))>0$ and $v_{B}(\alpha(\mathrm{q}), \beta(\mathrm{a}), \alpha(\mathrm{r}))<1$.

But $\mu_{B}(\alpha(\mathrm{q}), \beta(\mathrm{a}), \alpha(\mathrm{r}))=\vee\left\{\mu_{A}(\mathrm{q}, \mathrm{a}, \mathrm{t}) \mid \alpha(\mathrm{t})=\alpha(\mathrm{r})\right\}, v_{B}(\alpha(\mathrm{q}), \beta(\mathrm{a}), \alpha(\mathrm{r}))=\wedge\left\{v_{A}(\mathrm{q}, \mathrm{a}, \mathrm{t}) \mid \alpha(\mathrm{t})=\alpha(\mathrm{r})\right\}$.

Since $Q_{A}$ is a finite, there exists $t \in Q_{A}$ and $r \in Q_{B}$ such that $\alpha(\mathrm{t})=\alpha(\mathrm{r})$

and $\mu_{B}(\alpha(\mathrm{q}), \beta(\mathrm{a}), \alpha(\mathrm{r}))=\mu_{A}(\mathrm{q}, \mathrm{a}, \mathrm{t})>0$.

Suppose $\alpha(\mathrm{p})=\alpha(\mathrm{q})$, then $\mu_{A}(\mathrm{q}, \mathrm{a}, \mathrm{t})=\mu_{B}(\alpha(\mathrm{q}), \beta(\mathrm{a}), \alpha(\mathrm{r}))=\mu_{B}(\alpha(\mathrm{p}), \beta(\mathrm{a}), \alpha(\mathrm{r})) \geq \mu_{A}(\mathrm{p}, \mathrm{a}, \mathrm{r})$

and $v_{A}(\mathrm{q}, \mathrm{a}, \mathrm{t})=v_{B}(\alpha(\mathrm{q}), \beta(\mathrm{a}), \alpha(\mathrm{r}))=v_{B}(\alpha(\mathrm{p}), \beta(\mathrm{a}), \alpha(\mathrm{r})) \leq v_{A}(\mathrm{p}, \mathrm{a}, \mathrm{r})$.

Lemma 3.5 If the mapping $\beta: \Sigma \rightarrow \Gamma$ is extended as $\beta^{*}: \Sigma^{*} \rightarrow \Gamma^{*}$ by

1. $\beta^{*}(\varepsilon)=\beta(\varepsilon)=\varepsilon$

2. $\beta^{*}\left(\mathrm{a}_{1} \mathrm{a}_{2} \ldots . \mathrm{a}_{n}\right)=\beta\left(\mathrm{a}_{1}\right) \beta\left(\mathrm{a}_{2}\right) \ldots . \beta\left(\mathrm{a}_{n}\right), \mathrm{n} \geq 0, \mathrm{a}_{1}, a_{2} \ldots, a_{n} \in \Sigma$.

Then $\beta^{*}(\mathrm{xy})=\beta^{*}(\mathrm{x}) \beta^{*}(\mathrm{y}), \forall \mathrm{x}, \mathrm{y} \in \Sigma^{*}$.

Lemma 3.6 If $(\alpha, \beta)$ is a homomorphism. Then $\forall x \in \Sigma^{*}, p, q \in Q_{A}$,

1. $\mu_{A}^{*}(\mathrm{p}, \mathrm{x}, \mathrm{q}) \leq \mu_{B}^{*}\left(\alpha(\mathrm{p}), \beta^{*}(\mathrm{x}), \alpha(\mathrm{q})\right)$ and $v_{A}^{*}(\mathrm{p}, \mathrm{x}, \mathrm{q}) \geq v_{B}^{*}\left(\alpha(\mathrm{p}), \beta^{*}(\mathrm{x}), \alpha(\mathrm{q})\right)$

2. $i_{\mu_{A}}(\mathrm{p}) \leq i_{\mu_{B}}(\alpha(\mathrm{p}))$ and $i_{v_{A}}(\mathrm{p}) \geq i_{v_{B}}(\alpha(\mathrm{p})), \forall \mathrm{p} \in \mathrm{Q}_{A}$

3. $f_{\mu_{A}}(\mathrm{p}) \leq f_{\mu_{B}}(\alpha(\mathrm{p}))$ and $f_{v_{A}}(\mathrm{p}) \geq f_{v_{B}}(\alpha(\mathrm{p})), \forall \mathrm{p} \in \mathrm{Q}_{A}$

Proof: We prove (1) by induction on $|\mathrm{x}|=\mathrm{n}$ for $x \in \Sigma^{*}$. The result is trivial for $n=0$.

Assume that the result is true for all $x \in \Sigma^{*}$ such that $|x| \leq \mathrm{n}-1, \mathrm{n}>0$.

Let $|x|=\mathrm{n}, \mathrm{x}=$ ya where $y \in \Sigma^{*}, a \in \Sigma$ and $|y|=n-1$.

$$
\begin{aligned}
\mu_{A}^{*}(\mathrm{p}, \mathrm{x}, \mathrm{q}) & =\mu_{A}^{*}(\mathrm{p}, \mathrm{ya}, \mathrm{q}) \\
& =\vee\left\{\mu_{A}^{*}(\mathrm{p}, \mathrm{y}, \mathrm{r}) \wedge \mu_{A}(\mathrm{r}, \mathrm{a}, \mathrm{q}) \mid \mathrm{r} \in \mathrm{Q}_{A}\right\} \\
& \leq \vee\left\{\mu_{B}\left(\alpha(\mathrm{p}), \beta^{*}(\mathrm{y}), \alpha(\mathrm{r})\right) \wedge \mu_{B}(\alpha(\mathrm{r}), \beta(\mathrm{a}), \alpha(\mathrm{q})) \mid r \in Q_{A}\right\} \\
& \leq \vee\left\{\mu_{B}\left(\alpha(\mathrm{p}), \beta^{*}(\mathrm{y}), r^{\prime}\right) \wedge \mu_{B}\left(\mathrm{r}^{\prime}, \beta(\mathrm{a}), \alpha(\mathrm{q})\right) \mid r^{\prime} \in Q_{B}\right\} \\
& =\mu_{B}^{*}\left(\alpha(\mathrm{p}), \beta^{*}(\mathrm{y}) \beta(\mathrm{a}), \alpha(\mathrm{q})\right) \\
& =\mu_{B}^{*}\left(\alpha(\mathrm{p}), \beta^{*}(\mathrm{ya}), \alpha(\mathrm{q})\right) \\
& =\mu_{B}^{*}\left(\alpha(\mathrm{p}), \beta^{*}(\mathrm{x}), \alpha(\mathrm{q})\right)
\end{aligned}
$$

and

$$
\begin{aligned}
v_{A}^{*}(\mathrm{p}, \mathrm{x}, \mathrm{q}) & =v_{A}^{*}(\mathrm{p}, \mathrm{ya}, \mathrm{q}) \\
& =\wedge\left\{v_{A}^{*}(\mathrm{p}, \mathrm{y}, \mathrm{r}) \vee v_{A}(\mathrm{r}, \mathrm{a}, \mathrm{q}) \mid \mathrm{r} \in \mathrm{Q}_{A}\right\} \\
& \geq \wedge\left\{v_{B}\left(\alpha(\mathrm{p}), \beta^{*}(\mathrm{y}), \alpha(\mathrm{r})\right) \vee v_{B}(\alpha(\mathrm{r}), \beta(\mathrm{a}), \alpha(\mathrm{q})) \mid r \in Q_{A}\right\} \\
& \geq \wedge\left\{v_{B}\left(\alpha(\mathrm{p}), \beta^{*}(\mathrm{y}), r^{\prime}\right) \vee v_{B}\left(\mathrm{r}^{\prime}, \beta(\mathrm{a}), \alpha(\mathrm{q})\right) \mid r^{\prime} \in Q_{B}\right\} \\
& =v_{B}^{*}\left(\alpha(\mathrm{p}), \beta^{*}(\mathrm{y}) \beta(\mathrm{a}), \alpha(\mathrm{q})\right) \\
& =v_{B}^{*}\left(\alpha(\mathrm{p}), \beta^{*}(\mathrm{ya}), \alpha(\mathrm{q})\right) \\
& =v_{B}^{*}\left(\alpha(\mathrm{p}), \beta^{*}(\mathrm{x}), \alpha(\mathrm{q})\right)
\end{aligned}
$$

Thus the result is true for $|x|=n$, hence 1, 2 and 3 follows from the definition.

Theorem 3.7 Let $\mathcal{A}$ and $\mathcal{B}$ be two IFA-UM's with $\mathrm{L}_{\mathcal{A}}$ and $\mathrm{L}_{\mathcal{B}}$ be the intuitionistic fuzzy behavior of $\mathcal{A}$ and $\mathcal{B}$ respectively. Let $(\alpha, \beta): \mathcal{A} \rightarrow \mathcal{B}$ be a homomorphism. Then for all $\mathrm{x} \in \Sigma$, $\mathrm{L}_{\mu_{\mathcal{A}}}(\mathrm{x}) \leq \mathrm{L}_{\mu_{\mathcal{B}}}\left(\beta^{*}(\mathrm{x})\right)$ and $\mathrm{L}_{v_{\mathcal{A}}}(\mathrm{x}) \geq \mathrm{L}_{v_{\mathcal{B}}}\left(\beta^{*}(\mathrm{x})\right)$. 
Proof: Let $x \in \Sigma^{*}$,

Now, $\mathrm{L}_{\mu_{\mathcal{A}}}(\mathrm{x})=\vee\left\{\left\{i_{\mu_{A}}(\mathrm{p}) \wedge \mu_{A}^{*}(\mathrm{p}, \mathrm{x}, \mathrm{q}) \wedge \mathrm{f}_{\mu_{A}}(\mathrm{q}) \mid \mathrm{q} \in \mathrm{Q}_{A}\right\} \mid p \in Q_{A}\right\}$

and $\quad \mathrm{L}_{v_{\mathcal{A}}}(\mathrm{x})=\wedge\left\{\left\{i_{v_{A}}(\mathrm{p}) \vee v_{A}^{*}(\mathrm{p}, \mathrm{x}, \mathrm{q}) \vee \mathrm{f}_{v_{A}}(\mathrm{q}) \mid \mathrm{q} \in \mathrm{Q}_{A}\right\} \mid p \in Q_{A}\right\}$.

Since $Q_{A}$ is finite, there exists $r, s \in Q_{A}$ such that

$$
\begin{aligned}
\mathrm{L}_{\mu_{\mathcal{A}}}(\mathrm{x}) & =i_{\mu_{A}}(\mathrm{r}) \wedge \mu_{A}^{*}(\mathrm{r}, \mathrm{x}, \mathrm{s}) \wedge \mathrm{f}_{\mu_{A}}(\mathrm{~s}) \\
& \leq i_{\mu_{B}}(\alpha(\mathrm{r})) \wedge \mu_{B}^{*}\left(\alpha(\mathrm{r}), \beta^{*}(\mathrm{x}), \alpha(\mathrm{s})\right) \wedge f_{\mu_{B}}(\alpha(\mathrm{s})) \\
& \leq \vee\left\{\left\{i_{\mu_{B}}\left(\mathrm{r}^{\prime}\right) \wedge \beta_{B}^{*}\left(r^{\prime}, \beta^{*}(\mathrm{x}), \mathrm{s}^{\prime}\right) \wedge f_{\mu_{B}}\left(\alpha\left(\mathrm{s}^{\prime}\right) \mid \mathrm{s}^{\prime} \in \mathrm{Q}_{B}\right)\right\} \mid r^{\prime} \in Q_{B}\right\} \\
& =\mathrm{L}_{\mu_{\mathcal{B}}}\left(\beta^{*}(\mathrm{x})\right)
\end{aligned}
$$

and

$$
\begin{aligned}
\mathrm{L}_{v_{\mathcal{A}}}(\mathrm{x}) & =i_{v_{A}}(\mathrm{r}) \wedge v_{A}^{*}(\mathrm{r}, \mathrm{x}, \mathrm{s}) \wedge \mathrm{f}_{v_{A}}(\mathrm{~s}) \\
& \geq i_{v_{B}}(\alpha(\mathrm{r})) \vee v_{B}^{*}\left(\alpha(\mathrm{r}), \beta^{*}(\mathrm{x}), \alpha(\mathrm{s})\right) \vee f_{v_{B}}(\alpha(\mathrm{s})) \\
& \geq \wedge\left\{\left\{i_{v_{B}}\left(\mathrm{r}^{\prime}\right) \vee \beta_{B}^{*}\left(r^{\prime}, \beta^{*}(\mathrm{x}), \mathrm{s}^{\prime}\right) \vee f_{v_{B}}\left(\alpha\left(\mathrm{s}^{\prime}\right) \mid \mathrm{s}^{\prime} \in \mathrm{Q}_{B}\right)\right\} \mid r^{\prime} \in Q_{B}\right\} \\
& =\mathrm{L}_{v_{\mathcal{B}}}\left(\beta^{*}(\mathrm{x})\right) .
\end{aligned}
$$

Therefore, $\mathrm{L}_{\mu_{\mathcal{A}}}(\mathrm{x}) \leq \mathrm{L}_{\mu_{\mathcal{B}}}\left(\beta^{*}(\mathrm{x})\right)$ and $\mathrm{L}_{v_{\mathcal{A}}}(\mathrm{x}) \geq \mathrm{L}_{v_{\mathcal{B}}}\left(\beta^{*}(\mathrm{x})\right), \forall x \in \Sigma^{*}$.

Corollary 3.7.1 If $\beta$ is the identity map then $\mathrm{L}_{\mathcal{A}} \subseteq \mathrm{L}_{\mathcal{B}}$.

Lemma 3.8 If $(\alpha, \beta)$ is a strong homomorphism and $\alpha$ is bijective then $\forall p, q \in Q_{A}, x \in \Sigma^{*}$.

(i)

$$
\mu_{A}^{*}(\mathrm{p}, \mathrm{x}, \mathrm{q})=\mu_{B}^{*}\left(\alpha(\mathrm{p}), \beta^{*}(\mathrm{x}), \alpha(\mathrm{q})\right) \text { and } v_{A}^{*}(\mathrm{p}, \mathrm{x}, \mathrm{q})=v_{B}^{*}\left(\alpha(\mathrm{p}), \beta^{*}(\mathrm{x}), \alpha(\mathrm{q})\right) \text {, }
$$

(ii)

$$
i_{\mu_{A}}(\mathrm{p})=i_{\mu_{B}}(\alpha(\mathrm{p})) \text { and } i_{v_{A}}(\mathrm{p})=i_{v_{B}}(\alpha(\mathrm{p})) \text {, }
$$

(iii)

$$
f_{\mu_{A}}(\mathrm{p})=f_{\mu_{B}}(\alpha(\mathrm{p})) \text { and } f_{\mu_{A}}(\mathrm{p})=f_{\mu_{B}}(\alpha(\mathrm{p})) \text {. }
$$

Proof: Suppose $\alpha$ is one-one and onto.

Let $p, q \in Q_{A}, x \in \Sigma^{*}$. By induction on $|x|=n$. The result is trivial for $n=0$.

Suppose the result is true for $x \in \Sigma^{*},|x| \leq n-1$.

1. Let $|x|=\mathrm{n}, \mathrm{x}=$ ya where $y \in \Sigma^{*}, a \in \Sigma$ and $|y|=n-1$.

Then

$$
\begin{aligned}
\mu_{B}^{*}\left(\alpha(\mathrm{p}), \beta^{*}(\mathrm{x}), \alpha(\mathrm{q})\right) & =\mu_{B}^{*}\left(\alpha(\mathrm{p}), \beta^{*}(\mathrm{ya}), \alpha(\mathrm{q})\right) \\
& =\mu_{B}^{*}\left(\alpha(\mathrm{p}), \beta^{*}(\mathrm{y}) \beta(\mathrm{a}), \alpha(\mathrm{q})\right) \\
& =\vee\left\{\mu_{B}^{*}\left(\alpha(\mathrm{p}), \beta^{*}(\mathrm{y}), \mathrm{r}^{\prime}\right) \wedge \mu_{B}^{*}\left(\mathrm{r}^{\prime}, \beta(\mathrm{a}), \alpha(\mathrm{q})\right) \mid r^{\prime} \in Q_{B}\right\}
\end{aligned}
$$

and

$$
\begin{aligned}
v_{B}^{*}\left(\alpha(\mathrm{p}), \beta^{*}(\mathrm{x}), \alpha(\mathrm{q})\right) & =v_{B}^{*}\left(\alpha(\mathrm{p}), \beta^{*}(\mathrm{ya}), \alpha(\mathrm{q})\right) \\
& =v_{B}^{*}\left(\alpha(\mathrm{p}), \beta^{*}(\mathrm{y}) \beta(\mathrm{a}), \alpha(\mathrm{q})\right) \\
& =\wedge\left\{v_{B}^{*}\left(\alpha(\mathrm{p}), \beta^{*}(\mathrm{y}), \mathrm{r}^{\prime}\right) \vee v_{B}^{*}\left(\mathrm{r}^{\prime}, \beta(\mathrm{a}), \alpha(\mathrm{q})\right) \mid r^{\prime} \in Q_{B}\right\}
\end{aligned}
$$

Since $\alpha$ is onto, for $r^{\prime} \in Q_{B}$, there exists an $r \in Q_{A}$ such that $\alpha(\mathrm{r})=\mathrm{r}^{\prime}$.

Therefore,

$$
\begin{aligned}
\mu_{B}^{*}\left(\alpha(\mathrm{p}), \beta^{*}(\mathrm{x}), \alpha(\mathrm{q})\right) & =\vee\left\{\mu_{B}^{*}\left(\alpha(\mathrm{p}), \beta^{*}(\mathrm{y}), \alpha(\mathrm{r})\right) \wedge \mu_{B}(\alpha(\mathrm{r}), \beta(\mathrm{a}), \alpha(\mathrm{q})) \mid r \in Q_{A}\right\} \\
& =\vee\left\{\mu_{A}^{*}(\mathrm{p}, \mathrm{y}, \mathrm{r}) \wedge \mu_{A}(\mathrm{r}, \mathrm{a}, \mathrm{q}) \mid \mathrm{r} \in \mathrm{Q}_{A}\right\} \text { by induction } \\
& =\mu_{A}^{*}(\mathrm{p}, \mathrm{ya}, \mathrm{q}) \\
& =\mu_{A}^{*}(\mathrm{p}, \mathrm{x}, \mathrm{q})
\end{aligned}
$$

and 


$$
\begin{aligned}
v_{B}^{*}\left(\alpha(\mathrm{p}), \beta^{*}(\mathrm{x}), \alpha(\mathrm{q})\right) & =\wedge\left\{v_{B}^{*}\left(\alpha(\mathrm{p}), \beta^{*}(\mathrm{y}), \alpha(\mathrm{r})\right) \vee v_{B}^{*}(\alpha(\mathrm{r}), \beta(\mathrm{a}), \alpha(\mathrm{q})) \mid r \in Q_{A}\right\} \\
& =\wedge\left\{v_{A}^{*}(p, y, r) \vee v_{A}(r, a, q) \mid r \in Q_{A}\right\} \text { by induction } \\
& =v_{A}^{*}(\mathrm{p}, \mathrm{ya}, \mathrm{q}) \\
& =v_{A}^{*}(\mathrm{p}, \mathrm{x}, \mathrm{q})
\end{aligned}
$$

2. Let $p \in Q_{A}, i_{\mu_{B}}(\alpha(\mathrm{p}))=\vee\left\{i_{\mu_{A}}(\mathrm{r}) \mid \mathrm{r} \in \mathrm{Q}_{A}, \alpha(\mathrm{r})=\alpha(\mathrm{p})\right\}$ and

$$
i_{v_{B}}(\alpha(\mathrm{p}))=\wedge\left\{i_{v_{A}}(\mathrm{r}) \mid \mathrm{r} \in \mathrm{Q}_{A}, \alpha(\mathrm{r})=\alpha(\mathrm{p})\right\}
$$

Since $\alpha$ is one-one, $i_{\mu_{B}}(\alpha(\mathrm{p}))=i_{\mu_{A}}(\mathrm{p})$ and $i_{v_{B}}(\alpha(\mathrm{p}))=i_{v_{A}}(\mathrm{p})$.

3. Let $p \in Q_{A}, \mathrm{f}_{\mu_{B}}(\alpha(\mathrm{p}))=\vee\left\{f_{\mu_{A}}(\mathrm{r}) \mid \mathrm{r} \in \mathrm{Q}_{A}, \alpha(\mathrm{r})=\alpha(\mathrm{p})\right\}$

$$
f_{v_{B}}(\alpha(\mathrm{p}))=\wedge\left\{f_{v_{A}}(\mathrm{r}) \mid \mathrm{r} \in \mathrm{Q}_{A}, \alpha(\mathrm{r})=\alpha(\mathrm{p})\right\}
$$

Since $\alpha$ is one-one, $f_{\mu_{B}}(\alpha(\mathrm{p}))=f_{\mu_{A}}(\mathrm{p})$ and $f_{v_{B}}(\alpha(\mathrm{p}))=f_{v_{A}}(\mathrm{p})$.

Theorem 3.9 Let $\mathcal{A}$ and $\mathcal{B}$ be two IFA-UM's such that $\mathrm{L}_{\mathcal{A}}$ and $\mathrm{L}_{\mathcal{B}}$ be the intuitionistic fuzzy behavior of $\mathcal{A}$ and $\mathcal{B}$ respectively. Let $(\alpha, \beta)$ be a strong homomorphism and if $\alpha$ is bijective then $\mathrm{L}_{\mu_{\mathcal{A}}}(\mathrm{x})=$ $\mathrm{L}_{\mu_{\mathcal{B}}}\left(\beta^{*}(\mathrm{x})\right)$ and $\mathrm{L}_{v_{\mathcal{A}}}(\mathrm{x})=\mathrm{L}_{v_{\mathcal{B}}}\left(\beta^{*}(\mathrm{x})\right) \forall x \in \Sigma^{*}$.

Proof: Let $x \in \Sigma^{*}$,

$\mathrm{L}_{\mu_{B}}\left(\beta^{*}(\mathrm{x})\right)=\vee\left\{\left\{i_{\mu_{B}}\left(\mathrm{p}^{\prime}\right) \wedge \mu_{B}^{*}\left(\mathrm{p}^{\prime}, \beta^{*}(\mathrm{x}), \mathrm{q}^{\prime}\right) \wedge \mathrm{f}_{\mu_{B}}\left(\mathrm{q}^{\prime}\right) \mid \mathrm{q}^{\prime} \in \mathrm{Q}_{B}\right\} \mid p^{\prime} \in Q_{B}\right\}$

and

$\mathrm{L}_{v_{\mathcal{B}}}\left(\beta^{*}(\mathrm{x})\right)=\wedge\left\{\left\{i_{v_{B}}\left(\mathrm{p}^{\prime}\right) \wedge v_{B}^{*}\left(\mathrm{p}^{\prime}, \beta^{*}(\mathrm{x}), \mathrm{q}^{\prime}\right) \wedge \mathrm{f}_{v_{B}}\left(\mathrm{q}^{\prime}\right) \mid \mathrm{q}^{\prime} \in \mathrm{Q}_{B}\right\} \mid p^{\prime} \in Q_{B}\right\}$

Since $Q_{B}$ is finite there exists $r^{\prime}, s^{\prime} \in Q_{B}$ such that

$\mathrm{L}_{\mu_{\mathcal{B}}}\left(\beta^{*}(\mathrm{x})\right)=i_{\mu_{B}}\left(\mathrm{r}^{\prime}\right) \wedge \mu_{B}^{*}\left(\mathrm{r}^{\prime}, \beta^{*}(\mathrm{x}), \mathrm{s}^{\prime}\right) \wedge \mathrm{f}_{\mu_{B}}\left(\mathrm{~s}^{\prime}\right)$

and $\quad \mathrm{L}_{v_{\mathcal{B}}}\left(\beta^{*}(\mathrm{x})\right)=i_{v_{B}}\left(\mathrm{r}^{\prime}\right) \vee v_{B}^{*}\left(\mathrm{r}^{\prime}, \beta^{*}(\mathrm{x}), \mathrm{s}^{\prime}\right) \vee \mathrm{f}_{v_{B}}\left(\mathrm{~s}^{\prime}\right)$

Since $\alpha$ is onto, there exists $r, s \in Q_{A}$ such that $\alpha(\mathrm{r})=\mathrm{r}^{\prime}$ and $\alpha(\mathrm{s})=\mathrm{s}^{\prime}$.

Therefore,

$$
\begin{aligned}
& \mathrm{L}_{\mu_{\mathcal{B}}}\left(\beta^{*}(\mathrm{x})\right)=i_{\mu_{B}}(\mathrm{r}) \wedge \mu_{B}^{*}\left(r, \beta^{*}(\mathrm{x}), \alpha(\mathrm{s})\right) \wedge f_{\mu_{B}}(\alpha(\mathrm{s})), \\
& \mathrm{L}_{v_{\mathcal{B}}}\left(\beta^{*}(\mathrm{x})\right)=i_{v_{B}}(\mathrm{r}) \vee v_{B}^{*}\left(r, \beta^{*}(\mathrm{x}), \alpha(\mathrm{s})\right) \vee f_{v_{B}}(\alpha(\mathrm{s}))
\end{aligned}
$$

By Theorem 3.7 and Lemma 3.8, we get

$$
\mathrm{L}_{\mu_{\mathcal{A}}}(\mathrm{x})=\mathrm{L}_{\mu_{\mathcal{B}}}\left(\beta^{*}(\mathrm{x})\right) \text { and } \mathrm{L}_{v_{\mathcal{A}}}(\mathrm{x})=\mathrm{L}_{v_{\mathcal{B}}}\left(\beta^{*}(\mathrm{x})\right) \forall x \in \Sigma^{*} \text {. }
$$

\section{Admissible Relation}

Definition 4.1 Let $\mathcal{A}$ be an IFA-UM and $\sim$ be an equivalence relation on Q. Then $\sim$ is called an admissible relation if and only if for all $\mathrm{p}, \mathrm{q}, \mathrm{r} \in \mathrm{Q}, \forall \mathrm{a} \in \Sigma^{*}$, if $\mathrm{p} \sim \mathrm{q}, \mu_{\mathrm{A}}(\mathrm{p}, \mathrm{a}, \mathrm{r})>0$ and $v_{\mathrm{A}}(\mathrm{p}, \mathrm{a}, \mathrm{r})<1$, then there exists a $\mathrm{t} \in \mathrm{Q}$ such that $\mu_{\mathrm{A}}(\mathrm{p}, \mathrm{a}, \mathrm{r})=\mu_{\mathrm{A}}(\mathrm{q}, \mathrm{a}, \mathrm{t})$ and $v_{\mathrm{A}}(\mathrm{p}, \mathrm{a}, \mathrm{r})=v_{\mathrm{A}}(\mathrm{q}, \mathrm{a}, \mathrm{t}), \mathrm{t} \sim \mathrm{r}$.

Theorem 4.2 Let $\mathcal{A}$ be an IFA-UM and $\sim$ be an equivalence relation on $\mathrm{Q}$. Then $\sim$ is called an admissible relation if and only if for all $p, q, r \in Q, \forall x \in \Sigma^{*}$, if $p \sim q, \mu_{A}(p, x, r)>0$ and $v_{A}(p, x, r)<1$, then there exists at $\in \mathrm{Q}$ such that $\mu_{\mathrm{A}}(\mathrm{p}, \mathrm{x}, \mathrm{r})=\mu_{\mathrm{A}}(\mathrm{q}, \mathrm{x}, \mathrm{t})$ and $v_{\mathrm{A}}(\mathrm{p}, \mathrm{x}, \mathrm{r})=v_{\mathrm{A}}(\mathrm{q}, \mathrm{x}, \mathrm{t}), \mathrm{t} \sim \mathrm{r}$.

Proof: Suppose $\sim$ is an admissible relation on $\mathrm{Q}$. Let $\mathrm{p}, \mathrm{q} \in \mathrm{Q}$ be such that $p \sim q$.

Let $\mathrm{x} \in \Sigma^{*}{ }^{*} \mathrm{r} \in \mathrm{Q}$ be such that $\mu_{\mathrm{A}}^{*}(\mathrm{p}, \mathrm{x}, \mathrm{r})>0$ and $v_{\mathrm{A}}^{*}(\mathrm{p}, \mathrm{x}, \mathrm{r})<1$.

We prove the result by induction on $|\mathrm{x}|=\mathrm{n}$. The result is true for $\mathrm{n}=0$.

Assume that the result is true $\forall \mathrm{x} \in \Sigma^{*},|\mathrm{x}|<\mathrm{n}$. Let $|\mathrm{x}|=\mathrm{n}, \mathrm{x}=\mathrm{ya}$, where

$y \in \Sigma^{*}, a \in \Sigma,|y|=n-1$.

Let $p, q \in Q, p \sim q$ and $\mu_{A}^{*}(p, x, r)>0, v_{A}^{*}(p, x, r)<1$.

Therefore, $\mu_{\mathrm{A}}^{*}(\mathrm{p}, \mathrm{ya}, \mathrm{r})=\vee\left\{\mu_{\mathrm{A}}^{*}\left(\mathrm{p}, \mathrm{y}, \mathrm{q}_{1}\right) \wedge \mu_{\mathrm{A}}\left(\mathrm{q}_{1}, \mathrm{a}, \mathrm{r}\right) \mid \mathrm{q}_{1} \in \mathrm{Q}\right\}>0$ and

$v_{\mathrm{A}}^{*}(\mathrm{p}, \mathrm{ya}, \mathrm{r})=\wedge\left\{v_{\mathrm{A}}^{*}\left(\mathrm{p}, \mathrm{y}, \mathrm{q}_{1}\right) \vee v_{\mathrm{A}}\left(\mathrm{q}_{1}, \mathrm{a}, \mathrm{r}\right) \mid \mathrm{q}_{1} \in \mathrm{Q}\right\}<1$.

Since $Q$ is finite, there exists $s \in Q$ such that $\mu_{A}^{*}(p, y a, r)=\mu_{A}^{*}(p, s, r) \wedge \mu_{A}(s, a, r)>0$ 
and $v_{\mathrm{A}}^{*}(\mathrm{p}, \mathrm{ya}, \mathrm{r})=v_{\mathrm{A}}^{*}(\mathrm{p}, \mathrm{y}, \mathrm{s}) \bigvee v_{\mathrm{A}}(\mathrm{s}, \mathrm{a}, \mathrm{r})<1$, therefore, $\mu_{\mathrm{A}}^{*}(\mathrm{p}, \mathrm{y}, \mathrm{s})>0$ and $\mu_{\mathrm{A}}(\mathrm{s}, \mathrm{a}, \mathrm{r})>0, v_{\mathrm{A}}(\mathrm{s}, \mathrm{a}, \mathrm{r})<1$.

By induction, there exists $\mathrm{t}_{\mathrm{s}} \in \mathrm{Q}$ such that $\mu_{\mathrm{A}}^{*}(\mathrm{p}, \mathrm{y}, \mathrm{s})=\mu_{\mathrm{A}}^{*}\left(\mathrm{q}, \mathrm{y}, \mathrm{t}_{\mathrm{s}}\right)$ and $v_{\mathrm{A}}^{*}(\mathrm{p}, \mathrm{y}, \mathrm{s})=v_{\mathrm{A}}^{*}\left(\mathrm{q}, \mathrm{y}, \mathrm{t}_{\mathrm{s}}\right), \mathrm{t}_{\mathrm{s}} \sim \mathrm{s}$.

Now $\mu_{\mathrm{A}}(\mathrm{s}, \mathrm{a}, \mathrm{r})>0$ and $\mathrm{s} \sim \mathrm{t}_{\mathrm{s}}$ then there exists $\mathrm{t} \in \mathrm{Q}$ such that $\mu_{\mathrm{A}}(\mathrm{s}, \mathrm{a}, \mathrm{r})=\mu_{\mathrm{A}}\left(\mathrm{t}_{\mathrm{s}}, \mathrm{a}, \mathrm{t}\right)$ and

$v_{\mathrm{A}}(\mathrm{s}, \mathrm{a}, \mathrm{r})=v_{\mathrm{A}}\left(\mathrm{t}_{\mathrm{s}}, \mathrm{a}, \mathrm{t}\right), \mathrm{r} \sim \mathrm{t}$, therefore, $\mu_{\mathrm{A}}^{*}(\mathrm{p}, \mathrm{ya}, \mathrm{r})=\mu_{\mathrm{A}}^{*}\left(\mathrm{q}, \mathrm{y}, \mathrm{t}_{\mathrm{s}}\right) \wedge \mu_{\mathrm{A}}\left(\mathrm{t}_{\mathrm{s}}, \mathrm{a}, \mathrm{t}\right)$ and

$v_{\mathrm{A}}^{*}(\mathrm{p}, \mathrm{ya}, \mathrm{r})=v_{\mathrm{A}}^{*}\left(\mathrm{q}, \mathrm{y}, \mathrm{t}_{\mathrm{s}}\right) \vee v_{\mathrm{A}}\left(\mathrm{t}_{\mathrm{s}}, \mathrm{a}, \mathrm{t}\right)$.

Since $\mathcal{A}$ is an IFA-UM, the maximum or minimum will be arrived for any $\mathrm{r}^{\prime} \sim \mathrm{t}_{\mathrm{s}}$ only.

Therefore, $\mu_{A}^{*}(\mathrm{p}, \mathrm{ya}, \mathrm{r})=\vee\left\{\mu_{\mathrm{A}}^{*}\left(\mathrm{q}, \mathrm{y}, \mathrm{r}^{\prime}\right) \wedge \mu_{\mathrm{A}}\left(\mathrm{r}^{\prime}, \mathrm{a}, \mathrm{t}\right) \mid \mathrm{r}^{\prime} \in \mathrm{Q}\right\}=\mu_{\mathrm{A}}^{*}(\mathrm{q}, \mathrm{ya}, \mathrm{t}), \mathrm{r} \sim \mathrm{t}$.

i.e., $\quad \mu_{A}^{*}(p, y a, r)=\mu_{A}^{*}(q, x, t), r \sim t$ and

$v_{A}^{*}(p, y a, r)=\Lambda\left\{\mu_{A}^{*}\left(q, y, r^{\prime}\right) V \mu_{A}\left(r^{\prime}, a, t\right) \mid r^{\prime} \in Q\right\}=\mu_{A}^{*}(q, y a, t), r \sim t$.

i.e., $\quad v_{A}^{*}(p, y a, r)=v_{A}^{*}(q, x, t), r \sim t$.

Thus, the result is true for $|\mathrm{x}|=\mathrm{n}$. Hence the result.

Lemma 4.3 Let $\mathcal{A}$ be an IFA-UM and $\sim$ be an admissible relation on Q. Then there exists a fuzzy subset $\mu_{\mathrm{A}_{1}}: \mathrm{Q}_{1} \times \Sigma \times \mathrm{Q}_{1} \rightarrow[0,1]$, where $\mathrm{Q}_{1}=\mathrm{Q} / \sim$. Moreover, $\mu_{\mathrm{A}_{1}}$ is a fuzzy function of $\mathrm{Q}_{1} \times \Sigma \times[0,1]$ into $\mathrm{Q}_{1}$.

Proof: Let $\mathrm{q} \in \mathrm{Q}$ and $[\mathrm{q}]$ be the equivalence class of $\mathrm{q}$ i.e., $[\mathrm{q}]=\{\mathrm{p} \in \mathrm{Q} \mid \mathrm{q} \sim \mathrm{p}\}$.

Let $Q_{1}=Q / \sim=\{[q] \mid q \in Q\}$. Define $\mu_{\mathrm{A}_{1}}: Q_{1} \times \Sigma \times Q_{1} \rightarrow[0,1]$ and $v_{\mathrm{A}_{1}}: Q_{1} \times \Sigma \times Q_{1} \rightarrow[0,1]$

by $\quad \mu_{\mathrm{A}_{1}}([\mathrm{p}], \mathrm{a},[\mathrm{q}])=\mu_{\mathrm{A}}(\mathrm{p}, \mathrm{a}, \mathrm{q})$

$v_{\mathrm{A}_{1}}(([\mathrm{p}], \mathrm{a},[\mathrm{q}]))=v_{\mathrm{A}}(\mathrm{p}, \mathrm{a}, \mathrm{q}), \mathrm{r} \in[\mathrm{q}], \forall \mathrm{p} \in \mathrm{Q}, \mathrm{a} \in \Sigma$

Suppose $([p], a,[q])=\left(\left[p^{\prime}\right], a,\left[q^{\prime}\right]\right)$. Therefore $[p]=\left[p^{\prime}\right], a=b,[q]=\left[q^{\prime}\right]$ implies that $p \sim p^{\prime}$ and $q \sim q^{\prime}$. Let $\mu_{A}(\mathrm{p}, \mathrm{a}, \mathrm{r})>0, v_{A}(\mathrm{p}, \mathrm{a}, \mathrm{r})<1, \mathrm{r} \in[\mathrm{q}]$ and $\mathrm{p} \sim \mathrm{p}^{\prime}$. Since $\sim$ is an admissible relation on $Q$, there exists $t \in Q$ such that

$\mu_{A}(\mathrm{p}, \mathrm{a}, \mathrm{r})=\mu_{A}\left(\mathrm{p}^{\prime}, \mathrm{a}, \mathrm{t}\right), v_{A}(\mathrm{p}, \mathrm{a}, \mathrm{r})=v_{A}\left(\mathrm{p}^{\prime}, \mathrm{a}, \mathrm{t}\right), t \sim r$

$r \in[\mathrm{q}]$ implies that $r \in\left[\mathrm{q}^{\prime}\right]$ and so $\mathrm{t} \in\left[\mathrm{q}^{\prime}\right]$. By definition of $\mu_{A_{1}}$,

$\mu_{A}\left(\mathrm{p}^{\prime}, \mathrm{a}, \mathrm{t}\right)=\mu_{A_{1}}\left(\left[\mathrm{p}^{\prime}\right], \mathrm{a},\left[\mathrm{q}^{\prime}\right]\right), v_{A}\left(\mathrm{p}^{\prime}, \mathrm{a}, \mathrm{t}\right)=v_{A_{1}}\left(\left[\mathrm{p}^{\prime}\right], \mathrm{a},\left[\mathrm{q}^{\prime}\right]\right)$

From (1),(2),(3) and (4)

$\mu_{A_{1}}([\mathrm{p}], \mathrm{a},[\mathrm{q}])=\mu_{A_{1}}\left(\left[\mathrm{p}^{\prime}\right], \mathrm{b},\left[\mathrm{q}^{\prime}\right]\right)$ and $v_{A_{1}}([\mathrm{p}], \mathrm{a},[\mathrm{q}])=v_{A_{1}}\left(\left[\mathrm{p}^{\prime}\right], \mathrm{b},\left[\mathrm{q}^{\prime}\right]\right)$. Therefore, $\mu_{A_{1}}$ is well-defined.

We shall prove $\mu_{A_{1}}$ is a fuzzy function.

Let $\mu_{A_{1}}([\mathrm{p}], \mathrm{a},[\mathrm{q}])=\mu_{A_{1}}\left(\left[\mathrm{p}^{\prime}\right], \mathrm{a},\left[\mathrm{q}^{\prime}\right]\right)>0$ and $v_{A_{1}}([\mathrm{p}], \mathrm{a},[\mathrm{q}])=v_{A_{1}}\left([\mathrm{p}], \mathrm{a},\left[\mathrm{q}^{\prime}\right]\right)<1$. Therefore there exists $r, r^{\prime} \in Q$ such that $\mu_{A_{1}}([\mathrm{p}], \mathrm{a},[\mathrm{q}])=\mu_{A}(\mathrm{p}, \mathrm{a}, \mathrm{r}), v_{A_{1}}([\mathrm{p}], \mathrm{a},[\mathrm{q}])=v_{A}(\mathrm{p}, \mathrm{a}, \mathrm{r})$ and $r \sim q$,

$\mu_{A_{1}}\left([\mathrm{p}], \mathrm{a},\left[\mathrm{q}^{\prime}\right]\right)=\mu_{A}\left(\mathrm{p}, \mathrm{a}, \mathrm{r}^{\prime}\right), v_{A_{1}}\left([\mathrm{p}], \mathrm{a},\left[\mathrm{q}^{\prime}\right]\right)=v_{A}\left(\mathrm{p}, \mathrm{a}, \mathrm{r}^{\prime}\right)$ and $r^{\prime} \sim q^{\prime}$.

Therefore, $\mu_{A}(\mathrm{p}, \mathrm{a}, \mathrm{r})=\mu_{A}\left(\mathrm{p}, \mathrm{a}, \mathrm{r}^{\prime}\right), v_{A}(\mathrm{p}, \mathrm{a}, \mathrm{r})=v_{A}\left(\mathrm{p}, \mathrm{a}, \mathrm{r}^{\prime}\right)$. Since $\mathcal{A}$ is an IFA-UM, $r=r^{\prime}$, therefore $r \sim q$ and $r \sim q^{\prime}$. Hence $q \sim q^{\prime}$ and [q] $=\left[\mathrm{q}^{\prime}\right]$. Therefore, $\mu_{A_{1}}$ is a fuzzy function from $Q_{1} \times \Sigma \times[0,1]$ into $\mathrm{Q}_{1}$.

Definition 4.4 Let $\mathcal{A}=(\mathrm{Q}, \Sigma, \mathrm{A}, \mathrm{i}, \mathrm{f})$ be an IFA-UM and $\sim$ be an equivalence relation on $\mathrm{Q}$.

Let $Q_{1}=Q / \sim$. Define the IFA-UM $\mathcal{A}_{1}=\left(\mathrm{Q}_{1}, \Sigma, \mathrm{A}_{1}, \mathrm{i}_{1}, \mathrm{f}_{1}\right)$, where $\mu_{A_{1}}$ is a fuzzy subset $\mu_{A_{1}}: Q_{1} \times \Sigma \times Q_{1} \rightarrow[0,1]$ such that $\forall[\mathrm{p}],[\mathrm{q}] \in \mathrm{Q}_{1}, \mu_{A_{1}}([\mathrm{p}], \mathrm{a},[\mathrm{q}])=\mu_{A}(\mathrm{p}, \mathrm{a}, \mathrm{r}), \quad v_{A_{1}}([\mathrm{p}], \mathrm{a},[\mathrm{q}])=v_{A}(\mathrm{p}, \mathrm{a}, \mathrm{r}), \quad r \in[\mathrm{q}]$.

$i_{\mu_{\mu_{1}}}: Q_{1} \rightarrow[0,1], i_{v_{A 1}}: Q_{1} \rightarrow[0,1]$ such that

$i_{\mu_{A_{1}}}([\mathrm{p}])=\vee\left\{i_{\mu_{A_{1}}}(\mathrm{q}) \mid \mathrm{q} \in[\mathrm{p}]\right\}, i_{\nu_{A_{1}}}([\mathrm{p}])=\wedge\left\{i_{\nu_{A_{1}}}(\mathrm{q}) \mid \mathrm{q} \in[\mathrm{p}]\right\}$

$f_{\mu_{A_{1}}}: Q_{1} \rightarrow[0,1], f_{\nu_{A_{1}}}: Q_{1} \rightarrow[0,1]$ such that

$f_{\mu_{A_{1}}}([\mathrm{p}])=\vee\left\{f_{\mu_{A_{1}}}(\mathrm{q}) \mid \mathrm{q} \in[\mathrm{p}]\right\}, f_{\nu_{A_{1}}}([\mathrm{p}])=\wedge\left\{f_{\nu_{A_{1}}}(\mathrm{q}) \mid \mathrm{q} \in[\mathrm{p}]\right\}$.

Theorem 4.5 Let IFA-UM $\mathcal{A}$ and $\mathcal{A}_{1}$ be as in the definition 4.4. Then there exists a strong homomorphism from $\mathcal{A}$ to $\mathcal{A}_{1}$.

Proof: The proofs are straightforward.

\section{Conclusion}

In this paper, the algebraic nature of intuitionistic fuzzy finite automata under homomorphism and strong homomorphism of IFA-UM's is dealt with some results and illustrated with an example. Finally, the authors have made a humble beginning in this direction. However, the work highlights on the possibility of further research on fuzzyfying many more concepts in the context of IFA-UM. 


\section{References}

[1]. K.T. Atanassov, Intuitionistic fuzzy sets, Fuzzy Sets and Systems, vol. 20, no.1,(1986),87-96.

[2]. K.T. Atanassov, More on intuitionistic fuzzy sets, Fuzzy Sets and Systems, vol. 33, no.1,(1989a),37-46

[3]. K.T. Atanassov, New operations defined over the intuitionistic fuzzy sets, Fuzzy Sets Syst vol.61, no.2,(1994),137-142

[4]. P.Burillo ,H.Bustince, Vague sets are intuitionistic fuzzy sets, Fuzzy Sets Syst vol.79, (1996),403-405.

[5]. Y.B.Jun, Intuitionistic fuzzy finite state machines, J. Appl. Math. Comput. vol.17, no.1-2,(2005),109-120.

[6]. E.T. Lee, L.A. Zadeh, Note an fuzzy languages, Infom. Sci. vol. 1,(1969),421-434.

[7]. D.S. Malik and J.N. Mordeson, Fuzzy Automata and languages, theory and applications, $\{$ CRC $\}, 2002$.

[8]. T. Rajaretnam and S. Ayyaswamy, Fuzzy finite State Automaton with Unique Membership transition on an input Symbol, The journal of Combinatorial Mathematics and combinatorial computing, vol. 69,(2009),151--164.

[9]. Telesphor Lakra, A.Jeny Jordon, K. Jency Priya and T. Rajaretnam, Intuitionistic Fuzzy Finite Automata with Unique membership transitions, Proceedings of IEEE Xplore,(2014),103-107

[10]. E.S. Santos, Maximum automata, Inform Control, vol. 12,(1968),367-377.

[11]. W.G. Wee, and K.S. Fu, A formulation of fuzzy automata and its applications as a model of Learning systems, IEEE Trans. on Systems Science and Cybernetics, SSC,(1969),215-223.

[12]. L.A. Zadeh, Fuzzy sets\}, Inform Control, vol. 8,(1965),338-353 